\title{
MODELAGEM DA SÍNTESE ENZIMÁTICA DE ÉSTERES ETÍLICOS
}

\author{
M. L. HARTH ${ }^{1}$, J. H. DANTAS ${ }^{1}$, P. T. MARCHINI ${ }^{1}$, L. B. do NASCIMENTO ${ }^{1}$, F. F. de \\ MORAES ${ }^{1}$ e G. M. ZANIN ${ }^{1}$
}

\author{
${ }^{1}$ Universidade Estadual de Maringá, Departamento de Engenharia Química \\ E-mail para contato: matharth@gmail.com
}

\begin{abstract}
RESUMO - O interesse na produção de combustíveis alternativos cujos processos não possuem grande impacto ambiental remete ao uso da rota bioquímica para produção de biodiesel. No entanto, esta rota alternativa ainda não é compreendida totalmente. Em busca de tal compreensão, foram desenvolvidos dois modelos fenomenológicos (um que considerava a inibição por etanol e que outro, não) e ajustaram-se dados experimentais a eles. Os resultados mostraram que os intermediários de reação não se ajustaram muito bem, ao passo que o produto final e o substrato inicial se ajustaram melhor; sendo que o modelo sem inibição também ajustou-se levemente melhor aos dados do que o modelo com inibição.
\end{abstract}

\section{INTRODUÇÃO}

A produção de combustíveis alternativos tem atraído atenção do mercado mundial frente à necessidade de substituir as fontes convencionais oriundas de petróleo. A produção de ésteres etílicos pode ser conduzida por várias rotas, no entanto, a transesterificação de óleos vegetais destaca-se no meio industrial.

A transesterificação dos óleos vegetais consiste na quebra das ligações ésteres dos ácidos graxos ligados ao glicerol e seguida por uma condensação com um álcool de cadeia curta. Apesar do processo de produção de éster ser bem estabelecido por rotas químicas, a rota bioquímica, em especial a enzimática, ainda é uma possibilidade real diante dos bons rendimentos e potencial redução dos custos de produção associados, além de atender aos apelos de uma química mais limpa e sustentável.

No entanto, a melhoria e otimização deste processo ainda são fatores a serem estudados já que, por exemplo, o biocatalisador sofre inibição quando o álcool encontra-se em excesso no meio reacional (Maleki et al., 2013). Uma maneira simples, que não demanda grande esforço laboratorial, de ser feita tal otimização é desenvolvendo modelos fenomenológicos e usar softwares de ajustes de parâmetros (Ostroski et al., 2009). O presente trabalho possui como objetivo elaborar um modelo fenomenológico para produção de ésteres etílicos catalisada por lipases de Burkholderia cepacia livre utilizando como substratos óleo de canola e etanol. Também, deseja-se ajustar tal modelo a um conjunto de dados experimentais.

\section{METODOLOGIA}




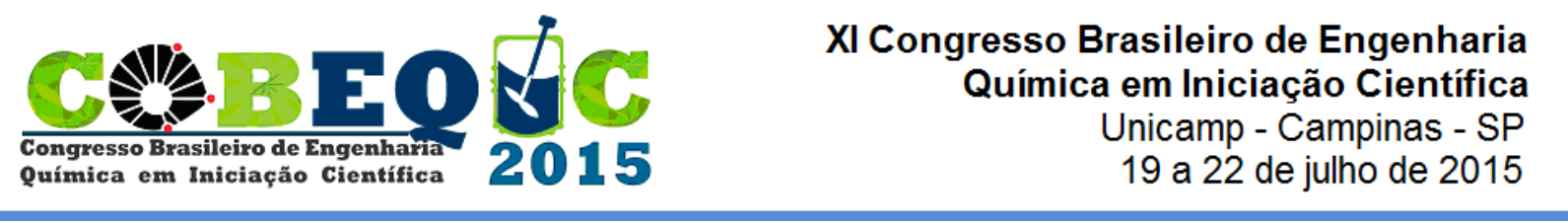

\subsection{Procedimento experimental}

Para os ensaios de síntese de ésteres etílicos utilizou-se óleo de canola e etanol como substratos, na proporção molar de 1:12, respectivamente. Utilizou-se lipase de Burkholderia cepacia (adquirida da Sigma-Aldrich) livre como biocatalisador, sendo adicionado ao meio reacional $10 \%(\mathrm{~m} / \mathrm{m})$ da mesma.

Tais ensaios foram conduzidos em reatores batelada em condição isotérmica a $40^{\circ} \mathrm{C}$ e mantidos em agitação constante por 72 horas. Condensadores foram utilizados para evitar evaporação de etanol, que foi adicionado em três momentos distintos (no início da reação, 12 e 24 horas depois), sendo que, cada injeção deste substrato consistia de um terço de seu volume total. Amostras foram retiradas em tempos pré-determinados. Para quantificar os triacilgliceróis, diacilgliceróis, monoacilgliceróis e ésteres etílicos utilizou-se cromatografia líquida de alta eficiência seguindo a metodologia de Holčapek et al. (2003).

\subsection{Modelagem matemática}

Para o desenvolvimento do modelo fenomenológico, consideraram-se apenas as reações que são responsáveis pela conversão do óleo de canola em ésteres etílicos, ou seja, sem levar em conta inibição de qualquer substrato ou produto. Tais reações estão descritas nas equações (1) a (3).

$$
\begin{aligned}
& \mathrm{Tri}+\mathrm{EtOH}+\mathrm{Ez} \square \quad \text { Tri } \square t \mathrm{EH} \square \mathrm{Ez} \square \quad \mathrm{Di}[\mathrm{EE} \sqsubset \mathrm{Ez} \square \quad \mathrm{Di}+\mathrm{EE}+\mathrm{Ez} \\
& \mathrm{Di}+\mathrm{EtOH}+\mathrm{Ez} \square \quad \mathrm{Di} \square \mathrm{EtOH} \square \mathrm{Ez} \square \quad \mathrm{M} \square \mathrm{EE} \square \mathrm{Ez} \square \quad \mathrm{M}+\mathrm{EE}+\mathrm{Ez} \\
& \mathrm{M}+\mathrm{EtOH}+\mathrm{Ez} \square \quad \mathrm{M} \square \mathrm{EtOH} \sqsubset \mathrm{Ez} \square \quad \mathrm{G} \sqsubset \mathrm{EE} \square \mathrm{Ez} \square \quad \mathrm{G}+\mathrm{EE}+\mathrm{Ez}
\end{aligned}
$$

em que Tri representa triacilglicerol; Di, diacilglicerol; M, monoacilglicerol; EtOH, etanol; EE, éster etílico; G, glicerol e Ez, enzima (o símbolo " $\sqcup$ " indica a formação do complexo ativo substratos-enzima ou produtos-enzima). Sabendo que todas as reações descritas anteriormente são elementares, usando a hipótese do estado pseudo-estacionário e realizando manipulações algébricas foram obtidas as seguintes equações diferenciais (Fogler, 2009).

$$
\begin{aligned}
& \frac{\mathrm{d}[\mathrm{Tri}]}{\mathrm{dt}}=\frac{-\mathrm{C}_{1}[\mathrm{Tri}][\mathrm{EtOH}]+\mathrm{C}_{2}[\mathrm{Di}][\mathrm{EE}]}{\alpha} \\
& \frac{\mathrm{d}[\mathrm{Di}]}{\mathrm{dt}}=\frac{\mathrm{C}_{1}[\mathrm{Tri}][\mathrm{EtOH}]-\mathrm{C}_{2}[\mathrm{Di}][\mathrm{EE}]-\mathrm{C}_{3}[\mathrm{Di}][\mathrm{EtOH}]+\mathrm{C}_{4}[\mathrm{M}][\mathrm{EE}]}{\alpha} \\
& \frac{\mathrm{d}[\mathrm{M}]}{\mathrm{dt}}=\frac{\mathrm{C}_{3}[\mathrm{Di}][\mathrm{EtOH}]-\mathrm{C}_{4}[\mathrm{M}][\mathrm{EE}]-\mathrm{C}_{5}[\mathrm{M}][\mathrm{EtOH}]+\mathrm{C}_{6}[\mathrm{G}][\mathrm{EE}]}{\alpha} \\
& \frac{\mathrm{d}[\mathrm{G}]}{\mathrm{dt}}=\frac{\mathrm{C}_{5}[\mathrm{M}][\mathrm{EtOH}]-\mathrm{C}_{6}[\mathrm{G}][\mathrm{EE}]}{\alpha}
\end{aligned}
$$




$$
\begin{aligned}
& \frac{\mathrm{d}[\mathrm{EE}]}{\mathrm{dt}}=\frac{\mathrm{C}_{1}[\mathrm{Tri}][\mathrm{EtOH}]-\mathrm{C}_{2}[\mathrm{Di}][\mathrm{EE}]-\mathrm{C}_{3}[\mathrm{Di}][\mathrm{EtOH}]+\mathrm{C}_{4}[\mathrm{M}][\mathrm{EE}]}{\alpha}+ \\
& +\frac{\mathrm{C}_{5}[\mathrm{M}][\mathrm{EtOH}]-\mathrm{C}_{6}[\mathrm{G}][\mathrm{EE}]}{\alpha}
\end{aligned}
$$$$
\frac{\mathrm{d}[\mathrm{EtOH}]}{\mathrm{dt}}=\frac{-\mathrm{C}_{1}[\mathrm{Tri}][\mathrm{EtOH}]+\mathrm{C}_{2}[\mathrm{Di}][\mathrm{EE}]-\mathrm{C}_{3}[\mathrm{Di}][\mathrm{EtOH}]+\mathrm{C}_{4}[\mathrm{M}][\mathrm{EE}]}{\alpha}+
$$$$
+\frac{-\mathrm{C}_{5}[\mathrm{M}][\mathrm{EtOH}]+\mathrm{C}_{6}[\mathrm{G}][\mathrm{EE}]}{\alpha}
$$

$$
\begin{aligned}
& \alpha=1+\mathrm{K}_{1}[\mathrm{Tri}][\mathrm{EtOH}]+\mathrm{K}_{2}[\mathrm{Di}][\mathrm{EE}]+\mathrm{K}_{3}[\mathrm{Di}][\mathrm{EtOH}]+\mathrm{K}_{4}[\mathrm{M}][\mathrm{EE}]+ \\
& +\mathrm{K}_{5}[\mathrm{M}][\mathrm{EtOH}]+\mathrm{K}_{6}[\mathrm{G}][\mathrm{EE}]
\end{aligned}
$$

Buscando tornar o modelo mais crível, foi feita uma tentativa de modelar a inibição por excesso de etanol no meio. Para isso, propôs-se a ocorrência de uma quarta reação, em que duas moléculas de etanol se unem, reversivelmente, ao sítio ativo formando um complexo enzimático inativo (EtOH[EtOH[Ez), conforme mostra a equação a seguir:

$$
\mathrm{EtOH}+\mathrm{EtOH}+\mathrm{Ez} \square \quad \mathrm{EtOH}\lceil\mathrm{EtOH} \square \mathrm{Ez}
$$

A inclusão desta nova reação altera o denominador $\alpha$ comum a todas as equações. Neste novo modelo, ele adquire o seguinte formato:

$$
\begin{aligned}
& \alpha=1+\mathrm{K}_{1}[\mathrm{Tri}][\mathrm{EtOH}]+\mathrm{K}_{2}[\mathrm{Di}][\mathrm{EE}]+\mathrm{K}_{3}[\mathrm{Di}][\mathrm{EtOH}]+\mathrm{K}_{4}[\mathrm{M}][\mathrm{EE}]+ \\
& +\mathrm{K}_{5}[\mathrm{M}][\mathrm{EtOH}]+\mathrm{K}_{6}[\mathrm{G}][\mathrm{EE}]+\mathrm{K}_{7}[\mathrm{EtOH}]^{2}
\end{aligned}
$$

Vale ressaltar que $\mathrm{C}_{\mathrm{i}}(\mathrm{i}=1,2, \ldots, 5,6)$ e $\mathrm{K}_{\mathrm{i}}(\mathrm{i}=1,2, \ldots, 6,7)$ são parâmetros dos modelos.

\subsection{Estimação dos parâmetros}

Por meio do ajuste do modelo aos dados experimentais é possível estimar os parâmetros cinéticos $\left(\mathrm{C}_{\mathrm{i}}\right.$ e $\left.\mathrm{K}_{\mathrm{i}}\right)$. Para isso, desenvolveu-se um algoritmo em MATLAB ${ }^{\circledR} 7.0$ cujo objetivo era minimizar a seguinte função-objetivo por meio da sub-rotina fmincon:

$$
\mathrm{S}=\sum_{\mathrm{i}} \sum_{\mathrm{t}=0}^{\mathrm{t}}\left[\mathrm{y}_{\mathrm{i}}(\mathrm{t})_{\exp }-\mathrm{y}_{\mathrm{i}}(\mathrm{t})_{\text {teo }}\right]^{2} / \mathrm{N}
$$

sendo $\mathrm{y}_{\mathrm{i}}(\mathrm{t})_{\exp }$ e $\mathrm{y}_{\mathrm{i}}(\mathrm{t})_{\text {teo }}$ as concentrações experimentais e calculadas pelo algoritmo do componente i no tempo t, respectivamente; e N, o número de concentrações experimentais obtidas. Para obter os valores de $\mathrm{y}_{\mathrm{i}}(\mathrm{t})_{\text {teo }}$, resolveu-se o sistema de equações diferenciais composto pelas Equações 4 a 10 e 13 utilizando o método numérico ode $23 s$ da biblioteca do MATLAB $^{\circledR}$ 7.0. Para que os parâmetros tivessem sentido físico, foi considerada a restrição de que todos os parâmetros deveriam ser positivos, ou seja, maiores ou igual a zero. 


\section{RESULTADOS E DISCUSSÃO}

Na Figura 1, encontra-se representada a resposta do modelo fenomenológico sem inibição e os dados experimentais. Analisando-a, os dados experimentais indicam que tanto o consumo de triacilglicerol como a produção de ésteres ocorrem rapidamente até 1000 minutos, sendo que, depois disso, a velocidade diminui consideravelmente. Já a concentração de diacilglicerol aumenta até atingir um máximo local e começa a diminuir, para logo em seguida atingir um novo máximo local. Este mesmo comportamento atípico foi observado, também, para monoacilglicerol. No entanto, sabe-se que a concentração de intermediários de reação, que é o caso destas espécies, deve aumentar até um valor máximo e, depois, diminuir até a concentração de equilíbrio. Acredita-se que tal anormalidade seja resultado da adição do etanol em etapas que aumentou a polaridade do meio e inibiu a catálise enzimática.

Figura 1 - Perfis de concentração de triacilglicerol, diacilglicerol, monoacilglicerol e éster etílico em função do tempo, sendo que as linhas cheias representam a resposta do modelo e os símbolos espaçados os valores experimentais, para o modelo sem inibição.

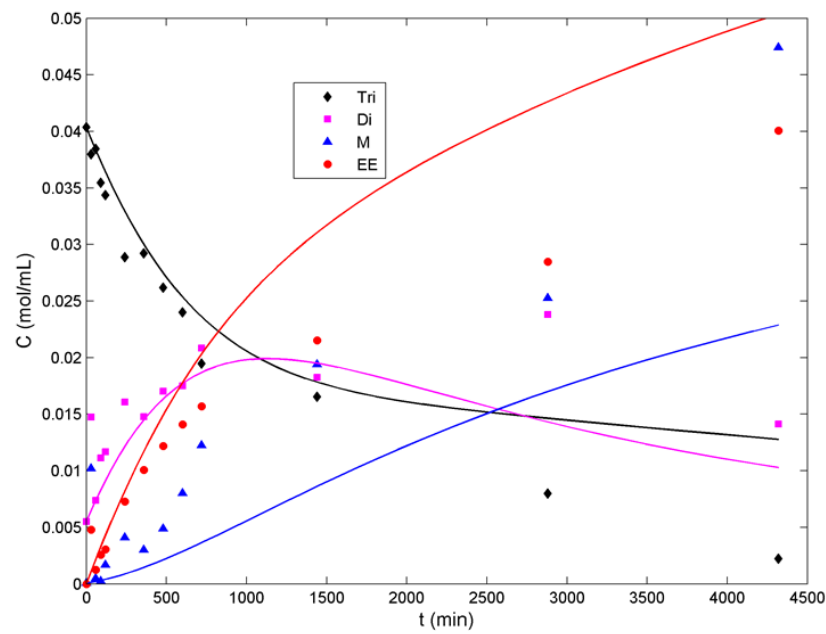

Agora, comparando o comportamento experimental com o previsto pelo modelo da mesma figura, nota-se que houve um bom ajuste para todas as espécies, com exceção do monoacilglicerol, até 1000 minutos. A partir deste momento, o modelo passou a superestimar a concentração de ésteres e triacilglicerol. Diferentemente do experimental, o perfil de concentração teórico de diacilglicerol apresentou um comportamento típico de intermediário de reação. $\mathrm{O}$ mesmo não pode ser afirmado sobre o de monoacilglicerol: aqui, ele se mostrou apenas crescente, seguindo a tendência experimental.

$\mathrm{Na}$ Figura 2, encontra-se a resposta do modelo com inibição, bem como os dados experimentais de concentração de triacilglicerol, diacilglicerol, monoacilglicerol e ésteres etílicos. Comparando as Figuras 1 e 2, nota-se que não houve mudanças significativas no perfil de concentração teórico das espécies. Realizando uma comparação criteriosa entre as Figuras 1 e 2, nota-se que os perfis de concentração teórico de triacilglicerol e diacilglicerol do modelo com inibição se afastam levemente dos dados experimentais. Tais observações remetem à ideia de que a modelagem da inibição por etanol não influencia muito a qualidade do ajuste do modelo 
Figura 2 - Perfis de concentração de triacilglicerol, diacilglicerol, monoacilglicerol e éster etílico em função do tempo para o modelo com inibição.

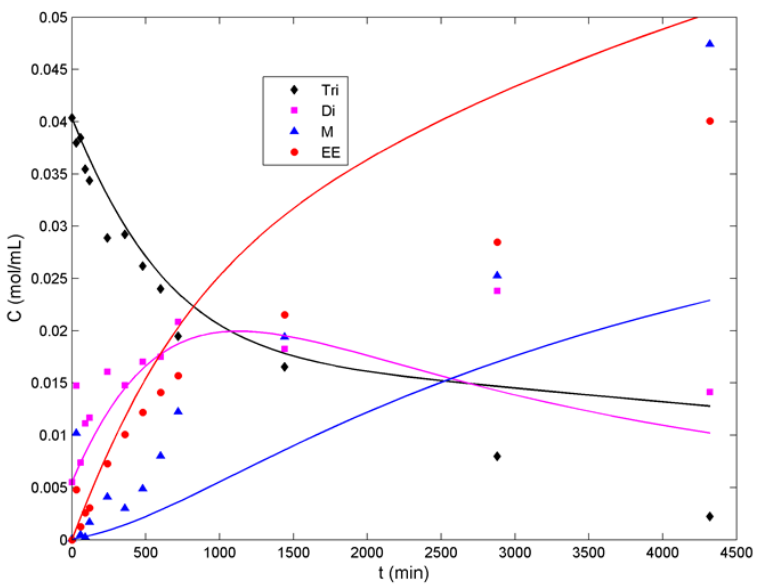

O algoritmo desenvolvido também respondia os valores dos parâmetros ajustados do modelo, que se encontram na Tabela 1 . Analisando-a atentamente, nota-se que a constante $\mathrm{C}_{1}$ é maior que $\mathrm{C}_{3}$ em ambos os modelos. Dessa forma, a conversão de triacilglicerol em diacilglicerol se procede mais rapidamente que a conversão do último em monoacilglicerol. Também, observa-se que $\mathrm{C}_{4}$ e $\mathrm{C}_{5}$ são iguais a zero. Estas duas constatações remetem ao acúmulo de diacilglicerol no meio reacional, explicando o comportamento crescente do perfil de concentração desta substância. Apesar de não possuir efeitos intensos, Gofferje et al. (2014) citam em seu trabalho que esta espécie, por conta da diferença de polaridade entre ela e a lipase, pode atuar como inibidor enzimático.

Tabela 1 - Valores calculados para as constantes dos modelos sem e com inibição.

\begin{tabular}{c|cc|cc}
\hline \multirow{2}{*}{$\mathrm{i}$} & \multicolumn{2}{|c|}{ Sem inibição } & \multicolumn{2}{c}{ Com inibição } \\
\cline { 2 - 5 } & $\mathrm{C}_{\mathrm{i}}(\mathrm{mL} / \mathrm{s} \cdot \mathrm{mol})$ & $\mathrm{K}_{\mathrm{i}}\left(\mathrm{mL}^{2} / \mathrm{mol}^{2}\right)$ & $\mathrm{C}_{\mathrm{i}}(\mathrm{mL} / \mathrm{s} . \mathrm{mol})$ & $\mathrm{K}_{\mathrm{i}}\left(\mathrm{mL}^{2} / \mathrm{mol}^{2}\right)$ \\
\hline 1 & 0,0018 & 0,4865 & 0,0021 & 0,2810 \\
2 & 0,0171 & 0,4947 & 0,0197 & 0,5022 \\
3 & 0,0008 & 0,6405 & 0,0009 & 0,1568 \\
4 & 0 & 0,4996 & 0 & 0,5022 \\
5 & 0 & 0,5949 & 0 & 0,2404 \\
6 & 1,1447 & 0,5001 & 0,8363 & 0,5000 \\
7 & - & - & - & 0,6597 \\
\hline
\end{tabular}

Com o objetivo de medir a qualidade do ajuste dos modelos, foi calculado o valor da função-objetivo. Estes valores encontram-se dispostos na Tabela 2. Nela, observa-se que, para ambos os modelos, este valor foi relativamente pequeno, considerando que a maioria dos pontos experimentais são menores que $0,05 \mathrm{~mol} / \mathrm{mL}$. Vale ressaltar que o valor da funçãoobjetivo do modelo sem inibição também foi levemente menor que o do modelo com inibição. Acredita-se que o motivo de não haver grandes diferenças na qualidade do ajuste entre os modelos é a possível solvatação do etanol pelo monoacilglicerol, que não foi considerado na elaboração do modelo. 
Tabela 2 - Valores da função-objetivo calculados para os modelos.

\begin{tabular}{cc}
\hline Modelo & $\mathrm{S}\left(\mathrm{mol}^{2} / \mathrm{mL}^{2}\right)$ \\
\hline Sem inibição & 0,0001451 \\
Com inibição & 0,0001452 \\
\hline
\end{tabular}

\section{CONCLUSÃO}

A partir dos resultados obtidos é possível afirmar que, até $1000 \mathrm{~min}$, ambos os modelos propostos se ajustaram bem às concentrações experimentais de triacilglicerol e éster etílico, enquanto que, para as demais, isto não foi observado. É possível que outro fenômeno, que não foi levado em consideração, esteja ocorrendo paralelamente, como, por exemplo, a solvatação de etanol pelo monoacilglicerol.

Também foi observado que o consumo de triacilglicerol é mais rápido que a conversão de diacilglicerol em monoacilglicerol, levando ao acúmulo no último no meio reacional, corroborando a hipótese da solvatação de etanol pelo mesmo. Outro fato notado é que o efeito da inibição por etanol no meio não foi tão intenso quanto era esperado, remetendo à ideia de que o modelo sem inibição se ajustou melhor.

\section{AGRADECIMENTOS}

Os autores agradecem à CAPES e ao CPNq pela bolsa e auxílio financeiro para realização da pesquisa.

\section{REFERENCIAS}

FOGLER, H. S. Elementos de Engenharia das Reações Químicas. 4. Rio de Janeiro: LTC, 2009.

GOFFERJE, G; STAEBLER, A.; HERFELLNER, T.; SCHWEIGGERT-WEISZ, U.; FLOETER, E. Kinetics of enzymatic esterification of glycerol and free fatty acids in crude Jatropha oil by immobilized lipase from Rhizomucor miehei. J Mol Catal BEnzym, v. 107, p. 1-7, 2014.

HOLČAPEK, M.; JANDERA, P.; ZDERADIČKA, P.; HRUBÁ, L. Characterization of triacylglycerol and diacylglycerol composition of plant oils using high-performance liquid chromatography-atmospheric pressure chemical ionization mass spectrometry. $J$ Chromatogr A, v. 1010, p. 195-215, 2003.

MALEKI, E.; AROUA, M. K.; SULAIMAN, N. M. N. Castor oil - a more suitable feedstock for enzymatic production of methyl esters. Fuel Process Technol, v. 112, p. 129-132, 2013.

OSTROSKI, I. C. BARROS, M. A. S. D.; SILVA, E. A.; DANTAS, J. H.; ARROYO, P. A.; LIMA, O. C. M. A comparative study for the ion exchange of Fe(III) and $\mathrm{Zn}$ (II) on zeolite NaY. J Hazard Mater, v. 161, p. 1404-1412, 2009. 